\title{
Impacto de la rehabilitación neurológica a través de la terapia de restricción inducida modificada en la mejora del apoyo social en usuarios con ataque cerebro vascular Impact of neurological rehabilitation through modified constraint induced therapy in improving social support patients with stroke
}

Arlette Doussoulin ${ }^{1}$, Jasim Najum², José L. Saiz ${ }^{3}$ y Fabiola Molina²

\begin{abstract}
Introduction: Access to rehabilitation in stroke patients is critical, considering the physical and psychological disabilities and generates social impact involved. Purpose: Evaluate the effectiveness of neurorehabilitation in collective mode, compared to the individual mode, in improving social support among patients with stroke, after application of the constraint induced of movement therapy. Methods: A randomized controlled trial of 36 patients who had a stroke were divided into two intervention groups. The independent variable was the implementation of collective or individual therapy for 3 hours for 10 consecutive days and the dependent variable was social perception evaluated by the Medical Outcomes Study-Social Support Survey (MOS-SSS) at baseline (pre-test) and end (post-test). Results: After controlling the pre-test scores, analysis of covariance revealed significant differences in favor of the collective mode in all post-test social support dimensions. Conclusions: Compared with the individual mode, the collective mode significantly improves several aspects of the patient's perceived social support, thus providing evidence of its clinical and practical effectiveness.
\end{abstract}

Keys words: Collective mode, Constraint-Induced Therapy, Neurorehabilitation, Social Support, Stroke.

Rev Chil Neuro-Psiquiat 2016; 54 (3): 187-197

Recibido: 30/11/2015

Aceptado: 04/07/2016

Los autores no presentan ningún tipo de conflicto de interés.

Financiamiento: proyecto Fondo Nacional de Investigación en Salud-FONIS SA13I20037.

1 Kinesiólogo, Msc. Motricidad Humana, PhD. Motricidad Humana, Académico Departamento de Pediatría y Cirugía Infantil, Universidad de La Frontera, Temuco, Chile.

2 Kinesiólogo, Hospital Hernán Henríquez Aravena, Temuco, Chile.

3 Psicólogo, PhD. Bioestadística, Académico Departamento de Psicología, Universidad de La Frontera, Temuco, Chile. 


\section{Introducción}

$\mathrm{L}^{2}$ a complejidad de una patología neurológica con alta prevalencia como el ataque cerebro vascular (ACV) lleva a que los usuarios presenten alteraciones físicas y psicosociales como depresión, fatiga y disminución en la participación y apoyo social $^{1-3}$. El apoyo social lo define Bowling como un proceso interactivo, en el cual el individuo obtiene ayuda emocional, instrumental y económica de la red social en la que se encuentra inmerso ${ }^{4}$.

Reeber (1992) plantea que el nivel de recuperación y los resultados obtenidos en la rehabilitación de los usuarios con discapacidad, pueden depender de la capacidad de la familia ${ }^{5}$ el entorno para proporcionar apoyo, considerando que este individuo se convierte en una carga importante para la fami$\mathrm{lia}^{6}$. Consecuentemente, el apoyo social y familiar es fundamental para el mantenimiento de la salud, la mejora de esta y la prevención de psicopatologías derivadas.

La rehabilitación, como proceso integral y multidisciplinario, debe estar orientado a apoyar al usuario y su familia, promoviendo la reintegración social, a través de estrategias terapéuticas efectivas y orientadas a mejorar la independencia funcional en actividades básicas e instrumentales de la vida diaria $^{7}$.

Diversas estrategias terapéuticas son utilizadas en este proceso; una de ellas es la Terapia de Restricción Inducida (TRI), técnica conductual aplicada en modalidad individual durante $6 \mathrm{~h}$. La TRI, basada en amplia evidencia científica ${ }^{8,9}$, ha demostrado ser efectiva en la rehabilitación de la extremidad superior induciendo una reorganización cortical en la zona lesionada ${ }^{10}$, permitiendo una mejor percepción del uso del brazo en actividades de la vida diaria (AVD) y transfiriendo estas mejoras al mundo real del usuario ${ }^{11}$. El propósito de esta terapia es mejorar la función sensitivo-motora de la extremidad superior, buscando reducir la discapacidad y promover la participación social ${ }^{7}$.

Con la finalidad de evaluar si una intervención en modalidad colectiva mejora el apoyo social en pacientes con ACV, fue modificado el protocolo original de la terapia (modalidad individual por
$6 \mathrm{~h}$ ), por una intervención grupal durante 3 h. La modalidad propuesta se fundamenta en los beneficios del trabajo en grupo, referidos a mejorar las redes de apoyo social, el logro del principio de universalidad y la cantidad de información, considerando que las fuentes de informaciones y experiencias se multiplican. Esta modalidad proveería un amplio espacio para el intercambio de ideas y apoyo emocional ${ }^{12}$, incentivando el proceso de rehabilitación ${ }^{13}$, y transfiriendo estas mejoras en la independencia funcional y participación en las $\mathrm{AVD}^{14}$.

El propósito de estudio es comparar la rehabilitación neurológica en modalidad colectiva versus la modalidad individual en el incremento del apoyo social en usuarios con ACV, posterior a la aplicación de la TRI.

\section{Material y Método}

\section{Diseño}

Se realizó un ensayo clínico aleatorizado simple ciego compuesto por 2 ramas paralelas (modalidad colectiva e individual), siendo el desenlace primario la mejora del apoyo social.

\section{Población y muestra}

Fueron identificados 120 usuarios pertenecientes a un centro comunitario de rehabilitación de la ciudad de Temuco, Chile; de ellos 76 no cumplieron los criterios de inclusión y 8 no aceptaron participar, quedando la muestra conformada por 36 usuarios (Figura 1), quienes firmaron el consentimiento informado previamente autorizado por el Comité de Ética de la Universidad de La Frontera.

El tamaño de la muestra se calculó considerando el número de sujetos con patologías neurológicas que ingresa mensualmente al centro de rehabilitación $(\mathrm{n}=40)$, basándose en un estimativo de la mínima diferencia significativa entre los grupos que corresponde a un $10 \%$, en relación con la variable dependiente. Se consideraron con 2 grupos, estimando un $10 \%$ de pérdida, un nivel de significancia del $5 \%$ y una potencia estadística de 0,80 .

Nueve fueron los criterios de inclusión: edad entre 30 y 80 años, presencia de un evento úni- 


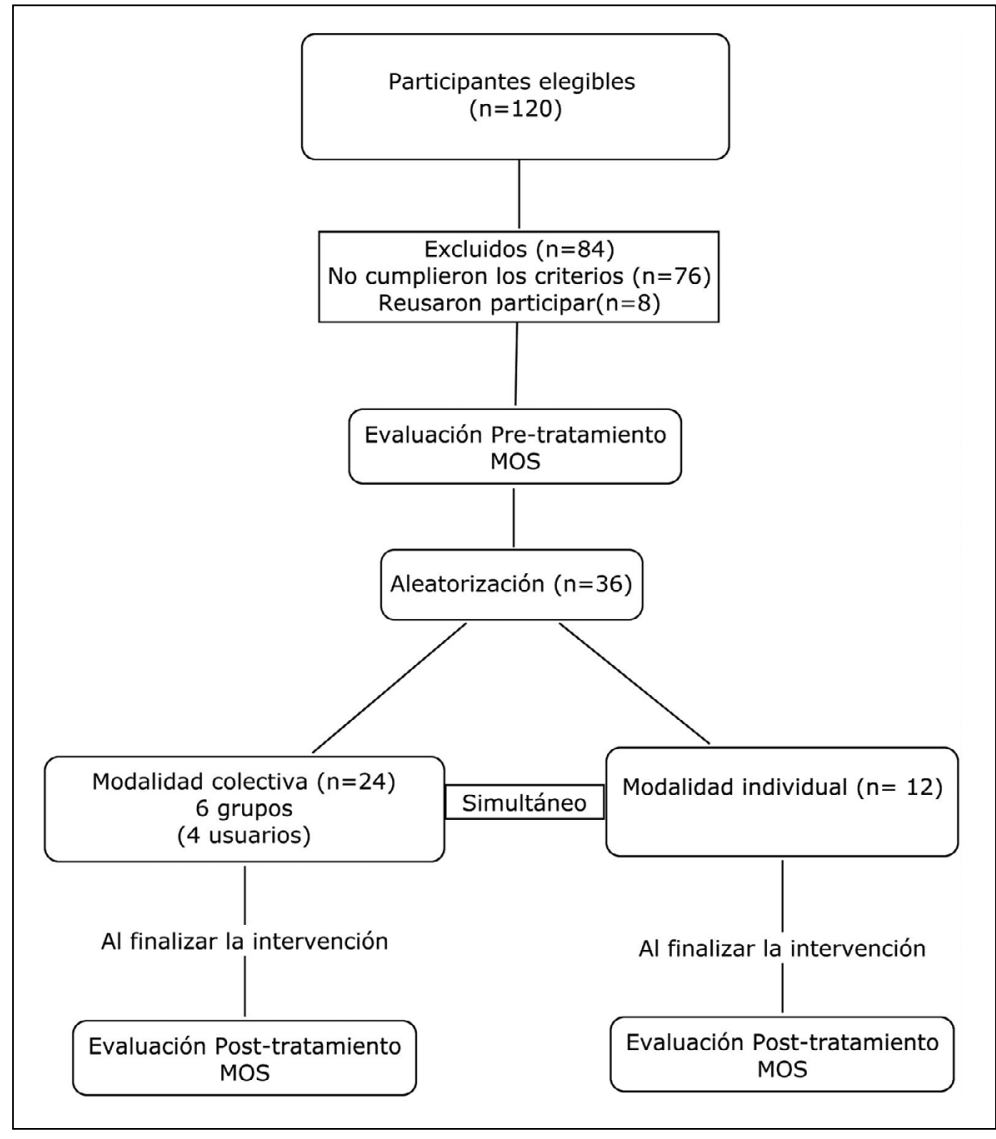

Figura 1. Flujograma de estudio, reclutamientoevaluación-intervención. co de ACV confirmado por TAC, una evolución mayor a 6 meses $^{15}$, capacidad de sentarse independientemente, una prueba funcional de $20^{\circ}$ de extensión de muñeca y $10^{\circ}$ de extensión de dedos ${ }^{16}$, obtención entre 5 y 14 puntos en la Escala NIHSS, menos de 2 puntos en la Escala de Ashworth Modificada, menos de 4 puntos en la Escala Visual Análoga y un puntaje mayor a 14 en la escala Minimental abreviada. Se excluyeron a los usuarios que presentaban compromiso sensorial (visualauditivo) o limitación ortopédica (uso de bastón).

\section{Procedimiento}

El reclutamiento fue realizado durante los meses de marzo a junio de 2014 por el coordinador del centro de rehabilitación. La información se recopiló a través de una ficha clínica e incluyó datos generales y registro de las variables de control: edad, género, dominancia, tipo de ACV y tiempo de evolución. Empleando la Medical Outcomes Study-Social Support Survey (MOS-SSS), un evaluador capacitado y sesgado realizó el pre-test una semana antes de la intervención y, manteniendo las mismas condiciones originales, el post-test al finalizar la intervención. El MOS-SSS es un instrumento ampliamente utilizado en la evaluación del apoyo social percibido en pacientes con enfermedades crónicas y ha mostrado adecuadas propiedades psicométricas. En este estudio se usó la versión del MOS-SSS validada en España y utilizada en Chile ${ }^{17,18}$.

El MOS-SSS está constituido por 20 ítems. El primer ítem cuantifica la red social preguntando por el número de amigos íntimos y familiares cercanos que tiene el evaluado. Los restantes ítems, que son respondidos en una escala tipo Likert desde 1 (nunca) hasta 5 (siempre), permiten computar un puntaje total de apoyo social global y puntajes en 4 
dimensiones específicas de apoyo: 1) Emocional/ Informacional, referida a la expresión de afecto y comprensión empática y a la guía y oferta de consejos e información (8 ítems; rango de puntajes: $8-40)$; 2) Instrumental, concerniente a la provisión de ayuda doméstica que pueda recibir la persona (4 ítems; rango: 4-20); 3) Interacción Social Positiva, referida a la disponibilidad de personas con las cuales poder salir, divertirse o distraerse ( 4 ítems; rango: 4-20) y 4) Afectivo, relativa a las expresiones de amor, afecto y empatía (3 ítems; rango: 3-15). El puntaje total tiene un rango de 19 a 95 . Mientras mayores son estos 5 puntajes, mayor es el nivel de apoyo social percibido correspondiente.

El protocolo de intervención TRIm, que corresponde a la variable independiente, fue aplicado en ambos grupos simultáneamente en modalidad colectiva e individual por $3 \mathrm{~h}$ diarias durante 10 días, por un kinesiólogo experto en la terapia y alumnos ayudantes. La intervención colectiva incluyó 6 grupos, cada uno compuesto por 4 usuarios $(\mathrm{n}=24)$ y los restantes 12 usuarios fueron expuestos a la modalidad individual. El protocolo aplicado, que cuenta con abundante soporte científico ${ }^{8,11}$, incluyó tres elementos principales en ambas modalidades: entrenamiento repetitivo orientado a la tarea, inducción al uso de la extremidad superior afectada y paquete de transferencia.
Entrenamiento repetitivo orientado a la tarea

Durante cada sesión, él o los pacientes recibieron ejercicios repetitivos que simulan actividades de la vida diaria, orientados al entrenamiento de la extremidad superior afectada. Los ejercicios se realizaron utilizando el modelado o shaping, técnica conductual cuyo objetivo es incrementar la cantidad y extensión de uso de la extremidad a través de la realización de tareas motoras. Para ello se contó con un banco de 30 tareas que involucran conjuntamente hombro, codo, muñeca y mano. Cada actividad funcional es practicada en diez series de diez intentos o durante un 60 a 120 segundos (Figura 2).

\section{Inducción al uso de la extremidad superior afectada}

Se realizó a través de la restricción motora de la extremidad superior indemne con un guante, que elimina la posibilidad de utilizar la mano durante la mayoría de las actividades funcionales, sobre todo cuando el kinesiólogo no está presente, es decir fuera del lugar de intervención. La meta es utilizar el guante el 70\% de las horas del día, evitando su uso en actividades como bañarse y cualquier actividad que genere peligro para el sujeto.

\section{Paquete de transferencia}

Una de las metas de la intervención es transferir

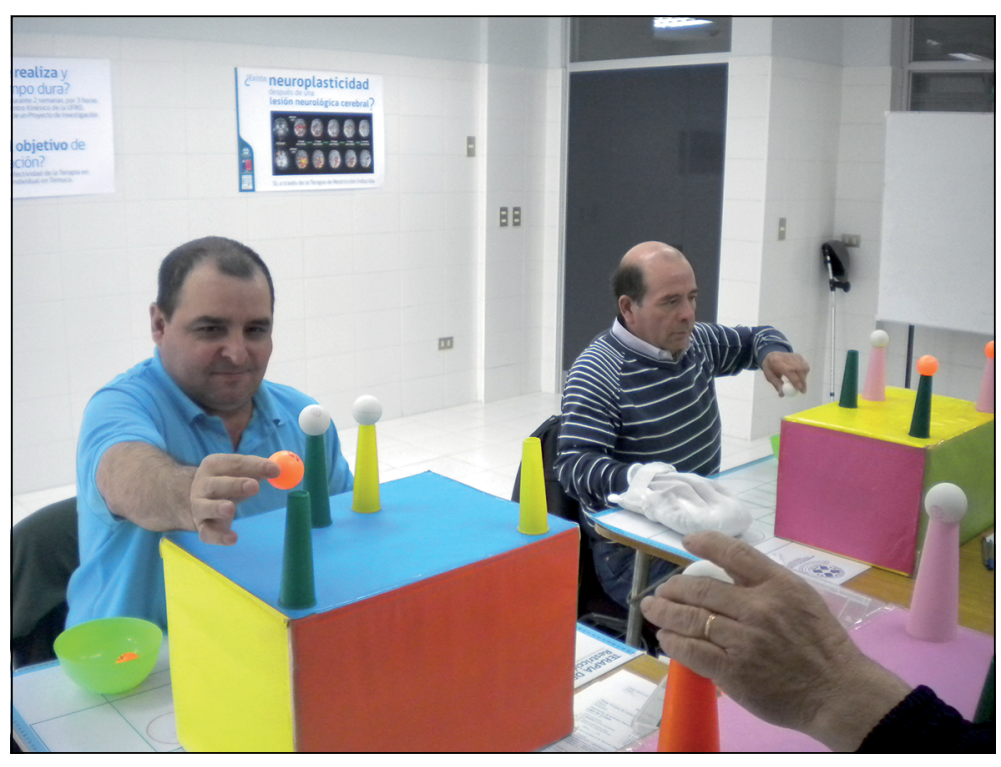

Figura 2. Ejercicio repetitivo orientado a la tarea, modalidad colectiva. 
los logros de la terapia al mundo real del usuario. Para alcanzar este objetivo se utilizó un conjunto de técnicas conductuales denominadas "paquete de transferencia" cuyo objetivo es hacer al usuario responsable de la adherencia a los requerimientos de la terapia, procurando que el usuario participe activamente de la intervención sin la supervisión constante del kinesiólogo, cuestión relevante en la vida diaria en la que este profesional no estará presente.

El procedimiento estándar de una sesión tipo, comenzaba con el ingreso de los participantes al lugar de terapia, luego se revisaba el Diario Casero, documento que describe las tareas (con y sin el guante) realizadas por el usuario en su casa durante el tiempo que no estuvo en terapia y que forma parte del paquete de transferencia. En la modalidad colectiva esta actividad fue compartida entre los participantes entregando la oportunidad para intercambiar información, dar y recibir retroalimentación y resolver inconvenientes. Posteriormente comenzaba la ejecución de ejercicios a través de tareas motoras específicas. Se realizaron 6 actividades definidas con anterioridad (usuarioskinesiólogo) las cuales simulaban actividades de la vida diaria e involucran movimientos de hombro, codo, muñeca y dedos. Cada actividad tiene sus propios requerimientos, parámetros, progresión y objetivo. Con la finalidad de evitar fatiga, aumento de tono y desmotivación, se realizaban elongaciones entre cada ejercicio y períodos de descanso. En la modalidad colectiva estos descansos permitían compartir experiencias entre los participantes. Los ejercicios es la actividad que utiliza el mayor porcentaje de horas durante la terapia, aproximadamente 70\%. La sesión finalizaba definiendo 5 tareas específicas a realizar en casa, utilizando el guante, con el objetivo de promover el uso del brazo débil fuera del lugar de terapia.

\section{Análisis de los datos}

El análisis incluyó a los 36 usuarios a través del software SPSS 15.0; no hubo pérdidas durante la intervención. Se evaluó la equivalencia inicial de los grupos en características sociodemográficas y clínicas relevantes, mediante la prueba de inde- pendencia $\chi^{2} \mathrm{o}$ análisis de varianza unifactorial intersujetos. Luego, a través del coeficiente alfa de Cronbach $^{19}$, se evaluó la consistencia interna de los puntajes del MOS-SSS. Finalmente, la comparación de las dos modalidades fue realizada mediante cinco análisis de covarianza unifactorial intersujetos, empleando las modalidades como variable independiente, cada uno de los cinco puntajes post-test del MOS-SSS como variable dependiente y los correspondientes puntajes pre-test del MOSS-SSS como covariables. Se empleó un nivel de significación de 0,05.

\section{Resultados}

\section{Equivalencia inicial de los grupos}

Como se observa en la Tabla 1, los dos grupos son inicialmente equivalentes en todas las variables sociodemográficas y clínicas consideradas, excepto en sexo. El grupo de la modalidad colectiva incluye relativamente más hombres $(75 \%)$ que el otro grupo (33\%).

\section{Consistencia interna del MOS-SSS}

En la Tabla 2 se presentan los índices alfa de cada puntaje del MOS-SSS según modalidad de intervención y tiempo de medición. De los veinte índices, 15 (75\%) fueron superiores a 0,70, valor calificado convencionalmente como mínimo aceptable $^{20}$. El rango de los 5 índices restantes fue 0,56-0,67. Considerando el reducido tamaño de los grupos y la pequeña cantidad de ítems en que se basan algunos de estos puntajes, los índices pueden ser considerados adecuados ${ }^{20}$. Por otra parte, los valores obtenidos son similares a los reportados por otros autores ${ }^{17,21}$.

\section{Efectividad de la modalidad de intervención en el incremento del apoyo social}

En relación a la red social, el promedio de amigos íntimos y familiares cercanos en la modalidad colectiva fue de 5,4 y en la modalidad individual fue 5,7 , diferencia que no es significativa $(\mathrm{p}>0,05)$. 
Tabla 1. Características sociodemográficas y clínicas de los participantes

\begin{tabular}{|c|c|c|c|}
\hline \multirow[t]{2}{*}{ Variable } & \multicolumn{2}{|c|}{ Modalidad } & \multirow[t]{2}{*}{ Prueba estadística } \\
\hline & Colectiva & Individual & \\
\hline Sexo, $f$ & & & $\chi^{2}(1)=5,84^{\star}$ \\
\hline Hombre & 18 & 4 & \\
\hline Mujer & 6 & 8 & \\
\hline Tipo ACV, $f$ & & & $\chi^{2}(6)=7,14$ \\
\hline Hemo intracerebral & 3 & 4 & \\
\hline Hemo intra-inespecífico & 0 & 1 & \\
\hline Intracraneal & 3 & 0 & \\
\hline Oclusión estenosis precerebral & 3 & 2 & \\
\hline Oclusión cerebral & 12 & 4 & \\
\hline ACV agudo & 2 & 0 & \\
\hline ACV indefinido & 1 & 1 & \\
\hline Edad, M (DE) & $58,33(10,38)$ & $48,75(18,60)$ & $\mathrm{F}(1,34)=0,51$ \\
\hline Espasticidad, M (DE) & $1,04(0,46)$ & $1,08(0,52)$ & $F(1,34)=0,06$ \\
\hline Dolor, M (DE) & $1,46(0,51)$ & $1,25(0,45)$ & $\mathrm{F}(1,34)=1,44$ \\
\hline Evolución, M (DE) & $25,63(23,52)$ & $39,00(33,51)$ & $\mathrm{F}(1,34)=1,94$ \\
\hline Mini mental, M (DE) & $16,58(1,53)$ & $16,75(2,01)$ & $F(1,34)=0,07$ \\
\hline
\end{tabular}

Nota. $\mathrm{f}=$ frecuencia, $\mathrm{M}=$ promedio, $\mathrm{DE}=$ desviación estándar. ${ }^{\star} \mathrm{p}<0,05$.

Tabla 2. Consistencia interna (alfa de Cronbach) de los puntajes de apoyo social por tiempo de medición (pre-test y post-test) y modalidad de tratamiento (colectiva e individual)

\begin{tabular}{|lcccc|}
\hline Dimensiones de apoyo & \multicolumn{2}{c}{ Puntajes pre-test } & \multicolumn{2}{c|}{ Puntajes post-test } \\
& Colectiva & Individual & Colectiva & Individual \\
Emocional & 0,88 & 0,97 & 0,89 & 0,94 \\
Instrumental & 0,64 & 0,75 & 0,67 & 0,70 \\
Interacción & 0,65 & 0,93 & 0,75 & 0,85 \\
Afectivo & 0,64 & 0,90 & 0,56 & 0,78 \\
Total & 0,89 & 0,97 & 0,90 & 0,96 \\
\hline
\end{tabular}

Como se exhibe en la Tabla 3, en la modalidad colectiva los promedios post-test de los cinco puntajes de apoyo social muestran un incremento en comparación con los respectivos promedios pre-test. En cambio, en la modalidad individual los promedios post-test muestran un descenso, excepto en Apoyo Instrumental donde surge un incremento. Al controlar las correspondientes evaluaciones pre-test, los análisis de covarianza revelaron que, en los cinco puntajes de MOS-SSS, la variable dependiente muestra diferencias significativas a favor de la modalidad colectiva. Según el coeficiente $\eta_{p}^{2}$ las modalidades explican desde un 18\% (en Apoyo Emocional) a un 45\% (en Apoyo Instrumental) de la varianza de los puntajes de apoyo social. 
Tabla 3. Efectos de la modalidad de tratamiento (colectiva e individual) en el apoyo social

\begin{tabular}{|lcccccccc|}
\hline Dimensiones de apoyo & \multicolumn{2}{c}{ Puntajes pre-test } & \multicolumn{2}{c}{ Puntajes post-test } & \multicolumn{2}{c|}{ ANCOVA } \\
& Colectiva & Individual & Colectiva & Individual & $F^{2}$ & $\eta_{p}^{2}$ \\
Emocional & $25,6(9,1)$ & $27,7(12,3)$ & $27,5(5,6)$ & $25,7(5,6)$ & $3,70^{*}$ & 0,18 \\
Instrumental & $16,8(3,8)$ & $16,4(3,8)$ & $18,5(3,4)$ & $17,4(2,3)$ & $13,73^{\star * *}$ & 0,45 \\
Interacción & $14,0(4,1)$ & $14,8(5,2)$ & $15,9(2,7)$ & $13,9(2,8)$ & $6,01^{\star *}$ & 0,28 \\
Afectivo & $12,5(2,5)$ & $12,9(3,8)$ & $13,7(1,7)$ & $11,5(1,8)$ & $12,81^{\star * *}$ & 0,44 \\
Total & $68,8(15,9)$ & $71,8(22,4)$ & $74,4(9,8)$ & $68,7(9,9)$ & $5,80^{\star *}$ & 0,26 \\
\hline
\end{tabular}

Nota. Puntajes son reportados como promedios (desviaciones típicas). Puntajes post-test están ajustados por los respectivos puntajes pre-test. ${ }^{\star} \mathrm{p}<0,05 .{ }^{* *} \mathrm{p}<0,01 .{ }^{* *} \mathrm{p}<0,001$.

\section{Discusión}

El estudio demuestra que la rehabilitación neurológica en modalidad colectiva, en comparación con la modalidad individual, produce incrementos significativos en distintos aspectos del apoyo social percibido en usuarios con ACV, posterior a la aplicación de 10 días de intervención con TRIm.

Si bien, existen numerosas estrategias de intervención que buscan rehabilitar a los usuarios con patologías de esta naturaleza ${ }^{22}$, la innovadora terapia de restricción inducida destaca, ya que posee amplia evidencia científica ${ }^{9}$, demostrando ser efectiva en la rehabilitación de la extremidad superior a través de ejercicios que simulan tareas funcionales que están directamente relacionadas con las demandas de la vida diaria ${ }^{23}$ e induciendo reorganización cortical en la zona lesionada ${ }^{10}$.

En relación a la modalidad de intervención "colectiva", son limitados los estudios que evalúan sus efectos sobre el apoyo social percibido ${ }^{2,24}$. De hecho, no hemos encontrado en la literatura estudios que evalúen el apoyo social en este tipo de usuarios y expuestos a una modalidad colectiva de intervención.

La intervención colectiva no puede definirse por oposición a la intervención individual, ya que también operan elementos interpersonales en la intervención individual. Toda persona se encuentra implicada en un contexto con el que mantiene una serie de relaciones, por lo cual cualquier actuación individual tiene presente la dimensión colectiva de la situación, facilitando la existencia de relaciones adecuadas con el entorno ${ }^{25}$. Por otro lado, en la intervención colectiva también operan elementos individuales, ya que los aspectos personales (capacidades, motivaciones, actitudes, etc.) están presentes en los procesos de trabajo colectivo.

Considerando los resultados obtenidos, la modalidad colectiva parece mejorar el apoyo social interviniendo en sus distintas dimensiones a través de la dinámica de trabajo generada por este tipo de intervención, las relaciones y redes sociales y las capacidades y potencialidades de las personas y de los grupos orientadas hacia la mejora colectiva.

La intervención colectiva, al proveer un contexto grupal, promueve el desarrollo de la autonomía personal, es decir, la capacidad individual para enfrentar y resolver problemas, aceptando sus falencias y afrontándolas directamente, permitiendo satisfacer necesidades humanas básicas como seguridad, afecto, sentido de pertenencia y autoestima $^{26}$.

Además, los usuarios en esta modalidad reciben una mayor cantidad de información y desde diversas fuentes, lo que abre un amplio espacio para el intercambio de ideas, sentimientos y otras vivencias. El contexto grupal ayuda al usuario a controlar la ansiedad, reduciendo una de las dimensiones importantes de la depresión y el estrés. Los grupos de soporte discuten temas de interés mutuo a consecuencia de la discapacidad que presentan. Adicionalmente, en esta modalidad se proporciona información específica acerca de cómo otros han 
salido adelante presentando el mismo problema y proporcionado a los sujetos la oportunidad de compartir sus emociones con personas con las mismas limitaciones ${ }^{27}$.

Asimismo, permite el logro de universalidad en cuanto a la apreciación de los problemas comunes, lo cual contribuye a que los sujetos estén menos centrados en sí mismos y a la vez se sientan desafiados en su proceso de rehabilitación.

Existe evidencia de que el apoyo social/emocional es un factor clave en el ajuste a la enfermedad y en la recuperación de la salud ${ }^{28}$. Este apoyo puede lograrse, por ejemplo, creando un clima de confianza, dando ánimo, permitiendo la libre expresión de los sentimientos y preocupaciones y el contacto social con otros. Otro proceso relacionado con el apoyo social/emocional es el proceso de comparación social en relación con la salud y el ajuste a la enfermedad ${ }^{29}$. Este proceso postula que las personas evalúan sus propias habilidades, actitudes y características mediante una comparación con otras personas similares. Cuando los individuos están temerosos por algo, buscarán afiliarse con otros sujetos que estén viviendo una situación similar para reducir su nivel de ansiedad.

En relación al apoyo familiar, la intervención colectiva promueve el soporte familiar en pacientes con enfermedades crónicas, estimulando el funcionamiento físico y emocional y favoreciendo la adherencia al tratamiento. Los miembros de la familia pueden no sólo recordar al paciente acerca de las actividades que necesita realizar, sino también participar de las actividades junto al paciente.

Durante la aplicación de la TRI colectiva, se vieron fortalecidas las cuatro dimensiones evaluadas por el MOS-SSS, destacando los apoyos instrumental y afectivo.

Esto puede ser explicado considerando que en las sesiones se generaba una dinámica de trabajo, apoyo mutuo y competitividad que llevaba a los participantes a desarrollar sus ejercicios con mayor motivación, dedicación y entrega, lo que se plasmaba en un mayor uso de su extremidad en situaciones cotidianas y posteriormente favorecía su participación social.

Se concluye que TRIm podría mejorar la rehabilitación de los usuarios en distintas esferas, siendo una de ellas el apoyo social. Altos niveles de apoyo social se asocian con una recuperación más rápida y extensa del estado funcional después de un $\mathrm{ACV}^{2}$.

Como limitaciones, se debe mencionar la no inclusión de otras variables potencialmente relevantes, exponiendo el trabajo al riesgo de un análisis insuficiente. Por ejemplo, este estudio no incluyó medidas de depresión siendo elevada su prevalencia en este tipo de usuarios ${ }^{30}$. Otra limitación resulta del reducido tamaño de la muestra que, aunque suficiente para detectar el efecto, no permite obtener estimadores más precisos ni explorar otras asociaciones en usuarios que presentan esta patología.

\section{Resumen}

Introducción: El acceso a rehabilitación en usuarios con ataque cerebro vascular es fundamental, considerando las discapacidades físicas y psicológicas que genera y el impacto social que conlleva. Objetivo: Evaluar la efectividad de la neurorehabilitación en modalidad colectiva, comparada con la modalidad individual, en la mejora del apoyo social en usuarios con ACV, después de la aplicación de la terapia de restricción inducida de movimiento. Método: Se realizó un ensayo clínico aleatorizado con 36 usuarios con ACV divididos en dos grupos de intervención. La variable independiente fue la implementación de la terapia en modalidad colectiva o individual por 3 horas diarias durante 10 días consecutivos y la variable dependiente fue el apoyo social percibido evaluado mediante la Medical Outcomes Study-Social Support Survey (MOS-SSS) antes (pretest) y después (post-test) de la intervención. Resultados: Controlando los puntajes pre-test, 
análisis de covarianza revelaron diferencias significativas a favor de la modalidad colectiva en todas las dimensiones post-test del apoyo social. Conclusiones: La rehabilitación en modalidad colectiva, en comparación con la modalidad individual, mejora significativamente diversos aspectos del apoyo social percibido del usuario, apoyando así su efectividad clínica y práctica.

Palabras clave: Apoyo social, ataque cerebro vascular, modalidad colectiva, rehabilitación neurológica, terapia de restricción inducida.

Anexo 1. CUESTIONARIO M.O.S

Nombre:

Puntaje Total:

Fecha:

Las siguientes preguntas se refieren al apoyo o ayuda de que Ud. dispone:

1. Aproximadamente, ¿Cuántos amigos íntimos o familiares cercanos tiene Ud.? (Personas con las que se encuentra a gusto y puede hablar acerca de todo lo que se le ocurre)

\section{Escriba el no de amigos y familiares \begin{tabular}{|l|l|}
\hline & \\
\hline
\end{tabular}}

La gente busca a otras personas para encontrar compañía, asistencia, u otros tipos de ayuda.

¿Con qué frecuencia dispone Ud. de cada uno de los siguientes tipos de apoyo cuando lo necesita? Marque con un círculo uno de los números de cada fila:

\begin{tabular}{|c|c|c|c|c|c|}
\hline & Nunca & $\begin{array}{l}\text { Pocas } \\
\text { veces }\end{array}$ & $\begin{array}{c}\text { Algunas } \\
\text { veces }\end{array}$ & $\begin{array}{l}\text { La mayoría } \\
\text { de veces }\end{array}$ & Siempre \\
\hline 2. Alguien que le ayude cuando tenga que estar en la cama & 1 & 2 & 3 & 4 & 5 \\
\hline 3. Alguien con quien puede contar cuando necesita $\mathrm{h}$ ar & 1 & 2 & 3 & 4 & 5 \\
\hline 4. Alguien que le aconseje cuando tenga problemas & 1 & 2 & 3 & 4 & 5 \\
\hline 5. Alguien que le lleve al médico cuando lo necesita & 1 & 2 & 3 & 4 & 5 \\
\hline 6. Alguien que le muestre amor y afecto & 1 & 2 & 3 & 4 & 5 \\
\hline 7. Alguien con quien pasar un buen rato & 1 & 2 & 3 & 4 & 5 \\
\hline 8. Alguien que le informe y le ayude a entender una situación & 1 & 2 & 3 & 4 & 5 \\
\hline $\begin{array}{l}\text { 9. Alguien en quien confiar o con quien hablar de sí mismo y sus } \\
\text { preocupaciones }\end{array}$ & 1 & 2 & 3 & 4 & 5 \\
\hline 10. Alguien que le abrace & 1 & 2 & 3 & 4 & 5 \\
\hline 11. Alguien con quien pueda relajarse & 1 & 2 & 3 & 4 & 5 \\
\hline 12. Alguien que le prepare la comida si no puede hacerlo & 1 & 2 & 3 & 4 & 5 \\
\hline 13. Alguien cuyo consejo realmente desee & 1 & 2 & 3 & 4 & 5 \\
\hline $\begin{array}{l}\text { 14. Alguien con quien hacer cosas que le sirvan para olvidar sus } \\
\text { problemas }\end{array}$ & 1 & 2 & 3 & 4 & 5 \\
\hline 15. Alguien que le ayude en sus tareas domésticas si está enfermo & 1 & 2 & 3 & 4 & 5 \\
\hline 16. Alguien con quien compartir sus temores y problemas más íntimos & 1 & 2 & 3 & 4 & 5 \\
\hline 17. Alguien que le aconseje cómo resolver sus problemas personales & 1 & 2 & 3 & 4 & 5 \\
\hline 18. Alguien con quien divertirse & 1 & 2 & 3 & 4 & 5 \\
\hline 19. Alguien que comprenda sus problemas & 1 & 2 & 3 & 4 & 5 \\
\hline 20. Alguien a quien amar y hacerle sentirse querido & 1 & 2 & 3 & 4 & 5 \\
\hline
\end{tabular}


Este Test, permite investigar 4 dimensiones de Apoyo:

1. Apoyo Emocional/Informacional: La expresión de afecto y comprensión empática, así como la guía y oferta de consejos e información.

Preguntas: 3-4-8-9-13-16-17-19

2. Apoyo Instrumental: La provisión de ayuda material que pueda recibir la persona. Preguntas: 2-5-12-15

3. La interacción social positiva: La disponibilidad de personas con las cuales poder salir, divertirse o distraerse. Preguntas: 7-11-14-18

4. Apoyo Afectivo: Las expresiones de amor y de afecto. Preguntas: 6-10-20

\begin{tabular}{|l|c|c|c|}
\hline Valores & Máximo & Mínimo & Medio \\
\hline Emocional & 40 & 8 & 24 \\
\hline Instrumental & 20 & 4 & 12 \\
\hline Interacción Social & 20 & 4 & 9 \\
\hline Afectivo & 15 & 3 & 57 \\
\hline Indice Global & 95 & 19 & 12 \\
\hline
\end{tabular}

El índice global de Apoyo Social se obtiene sumando los 19 ítems.

El Apoyo Social es Escaso cuando el Índice es inferior a 57 puntos.

Habrá Falta de Apoyo Emocional cuando la puntuación sea menor a 24.

Habrá Falta de Apoyo Instrumental cuando la puntuación sea menor a 12.

Habrá Falta de Interacción Social cuando la puntuación sea menor a 12.

Habrá Falta de Apoyo Afectivo cuando la puntuación sea menor a 9.

\section{Referencias bibliográficas}

1. Daniel K, Wolfe CD, Busch MA, McKevitt C. What are the social consequences of stroke for workingaged adults? A systematic review. Stroke 2009; 40 (6): e431-40.

2. Kumar A. Quality of life and psychosocial problems of persons after stroke. International Journal of Advanced Research 2015; 3 (5): 166-73.

3. Naranjo R, Bermúdez Y. Valor de la red de apoyo social en la evolución del hemipléjico. Rev Neurol 2002; 34: 998.

4. Chiu CY, Li YJ, Huang Y. Size-controlled synthesis of Pd nanocrystals using a specific multifunctional peptide. Nanoscale 2010; 2 (6): 927-30.

5. Reeber BJ. Evaluating the effects of a family education intervention. Rehabil Nurs 1992; 17 (6): 332-6.

6. Ski C, O'Connell B. Stroke: the increasing complexity of carer needs. J Neurosci Nurs 2007; 39 (3): 172-9.

7. Dromerick AW, Lang CE, Birkenmeier R, Hahn
MG, Sahrmann SA, Edwards DF. Relationships between upper-limb functional limitation and self-reported disability 3 months after stroke. J Rehabil Res Dev 2006; 43 (3): 401-8.

8. Peurala SH, Kantanen MP, Sjogren T, Paltamaa J, Karhula M, Heinonen A. Effectiveness of constraint-induced movement therapy on activity and participation after stroke: a systematic review and meta-analysis of randomized controlled trials. Clin Rehabil 2012; 26 (3): 209-23.

9. Kwakkel G, Veerbeek JM, van Wegen EE, Wolf SL. Constraint-induced movement therapy after stroke. Lancet Neurol 2015; 14 (2): 224-34.

10. Sawaki L, Butler AJ, Leng X, Wassenaar PA, Mohammad YM, Blanton S, et al. Differential patterns of cortical reorganization following constraintinduced movement therapy during early and late period after stroke: A preliminary study. Neuro Rehabilitation 2014; 35 (3): 415-26.

11. Taub E, Uswatte G, Bowman MH, Mark VW, Delgado A, Bryson C, et al. Constraint-induced 
movement therapy combined with conventional neurorehabilitation techniques in chronic stroke patients with plegic hands: a case series. Arch Phys Med Rehabil 2013; 94 (1): 86-94.

12. Sadock BJ, Kaplan HI, Sadock VA. Concise TextBook of clinical psychiatry: behavioral sciences/clinical psychiatry. Philadelphia: Lippincott Williams \& Wilkins., 2007.

13. Bandura A. A social learning theory. Englewood Cliffs, NJ: Prentice Hall. NJ: Englewood Cliffs, Prentice Hall., 1977.

14. Wu CY, Chen YA, Lin KC, Chao CP, Chen YT. Constraint-induced therapy with trunk restraint for improving functional outcomes and trunkarm control after stroke: a randomized controlled trial. Phys Ther 2012; 92 (4): 483-92.

15. Rijntjes M, Hobbeling V, Hamzei F, Dohse S, Ketels G, Liepert J, et al. Individual factors in constraintinduced movement therapy after stroke. Neurorehabil Neural Repair 2005; 19 (3): 238-49.

16. Wolf S, Miller J, Taub E, Uswatte G, Morris D, Giuliani C, et al. Effect of constraint-induced movement therapy on upper extremity function 3 to 9 months after stroke: the EXCITE randomized clinical trial. JAMA 2006; 296 (17): 2095-104.

17. Zheng HR, Nugent C, McCullagh P, Huang Y, Zhang SM, Burns W, et al. Smart self management: assistive technology to support people with chronic disease. J Telemed Telecare 2010; 16 (4): 224-27.

18. Shang Y, Huang Y, Huang HD, Bai C, Dong YC, Zhao LJ, et al. Removal of Glomus Tumor in the Lower Tracheal Segment with a Flexible Bronchoscope: Report of Two Cases. Internal Med 2010; 49 (9): 865-69.

19. Streiner DL. Being inconsistent about consistency: when coefficient alpha does and doesn't matter. J Pers Assess 2003; 80 (3): 217-22.

Correspondencia:

Arlette Doussoulin S. Hoschtetter 405 Temuco, Chile.

Teléfono: 56-45-2732411 / Fax: 56-45-2744308

arlette.doussoulin@ufrontera.cl
20. Streiner DL. Starting at the beginning: an introduction to coefficient alpha and internal consistency. J Pers Assess 2003; 80 (1): 99-103.

21. Chiou M, Huang Y. NP-anaphora in Modern Greek: A partial neo-Gricean pragmatic approach. J Pragmatics 2010; 42 (7): 2036-57.

22. Urton M, Kohia M, Davis J, Neill M. Systematic literature review of treatment interventions for upper extremity hemiparesis following stroke. Occup Ther Int 2007; 14 (1): 11-27.

23. Doussoulin A, Saiz J, Rivas R. Efectividad de la terapia de restricción inducida modificada en la recuperación de la cantidad y calidad de movimiento de la extremidad superior después de una enfermedad vascular cerebral aguda. Rev Mex Neuroci 2015; 4: 1-13.

24. Glass TA, Matchar DB, Belyea M, Feussner JR. Impact of social support on outcome in first stroke. Stroke 1993; 24 (1): 64-70.

25. De Robertis C, Pascal H. La intervención colectiva en el trabajo social: la acción con grupos y comunidades. $1^{\circ}$ ed. ed: Lumen Grupo Editorial, 1994.

26. Taylor SE. Health psychology. $8^{\circ}$ ed. ed. New York: McGraw Hill, 2011.

27. Gottlieb BH. Social Networks and Social Support: An Overview of Research, Practice, and Policy Implications. Health Educ Behav 1985; 12: 5-22.

28. Hatchett L, Friend R, Symister P, Wadhwa N. Interpersonal expectations, social support, and adjustment to chronic illness. Journal of Personality \& Social Psychology 1997; 73 (3): 560-73.

29. Suls JM, Wheeler L. Handbook of social comparison: theory and research. New York: Springer, 2000.

30. Schepers V, Post M, Visser-Meily A, van de Port I, Akhmouch M, Lindeman E. Prediction of depressive symptoms up to three years post-stroke. J Rehabil Med 2009; 41 (11): 930-5. 